Revue

d'ethnoécologie
Revue d'ethnoécologie

$20 \mid 2021$

Un siècle d'ethno-machins : le centenaire de la Revue de Botanique Appliquée

\title{
La Flora Común es la Flora Medicinal
}

Conocimiento Popular y la Herbolaria Tzeltal-Tzotzil de Los Altos de Chiapas, México

La flore commune est la flore médicinale: Savoir populaire et herboristerie Tzeltal-Tzotzil des Hautes-Terres du Chiapas, Méxique

The common flora is the medicinal flora: Folkknowledge and the Tzeltal-Tzotzil herbolaria

\section{Brent Berlin and Elois Ann Berlin}

\section{OpenEdition}

\section{Journals}

Electronic version

URL: https://journals.openedition.org/ethnoecologie/8436

DOI: 10.4000/ethnoecologie.8436

ISSN: 2267-2419

Publisher

Laboratoire Éco-anthropologie

\section{Electronic reference}

Brent Berlin and Elois Ann Berlin, "La Flora Común es la Flora Medicinal", Revue d'ethnoécologie [Online], 20 | 2021, Online since 31 December 2021, connection on 06 March 2022. URL: http://

journals.openedition.org/ethnoecologie/8436 ; DOI: https://doi.org/10.4000/ethnoecologie.8436

This text was automatically generated on 6 March 2022.

\section{(†)}

Revue d'ethnoécologie est mis à disposition selon les termes de la licence Creative Commons Attribution - Pas d'Utilisation Commerciale - Pas de Modification 4.0 International. 


\title{
La Flora Común es la Flora Medicinal
}

\author{
Conocimiento Popular y la Herbolaria Tzeltal-Tzotzil de Los Altos de \\ Chiapas, México \\ La flore commune est la flore médicinale: Savoir populaire et herboristerie \\ Tzeltal-Tzotzil des Hautes-Terres du Chiapas, Méxique \\ The common flora is the medicinal flora: Folkknowledge and the Tzeltal-Tzotzil \\ herbolaria
}

\section{Brent Berlin and Elois Ann Berlin}

« Hace décadas, Don Antonio Pérez Méndez ha utilizado los secretos centenarios de sus antipasados como tratamientos y curaciones de

las enfermedades comunes de su comunidad.

Ahora, interés reciente en estos 'secretos' medicinales por « conglomerantes » de investigación y por universidades bastante bien

financiados del Norte pretenden robar esta herencia (Caplan $2000: 1$ ).

\section{Introducción}

Este ponencia se hace distinción definitiva entre el conocimiento especial o secreto y el conocimiento popular de las especies de plantas medicinales que forman la base de la herbolaria empírica de los tzeltales y tzotziles de los Altos de Chiapas. Pretendemos demostrar que la herbolaria maya consiste principalmente de la flora común de la región. Además, estas especies están reconocidas por la población en general, no solamente por unas especialistas o individuos que se identifican como médicos tradicionales. Podemos concluir que la herbolaria tzeltal-tzotzil es, entonces, la medicina maya popular en el mismo sentido que podemos hablar de la medicina mexicana 
popular. La herbolaria de los mayas no consiste en información sagrada ni conocimiento secreto. El sistema es público y merece estudios antropológicos, etnobotánicos, etnomédicos, y de laboratorio, siempre en estrecha colaboración con las comunidades mayas, sobre métodos de preparación y administración, bioactividad, toxicidad, y control de calidad.

\section{Objetivos}

2 Presentamos datos orginales que demuestran que la flora medicinal de los mayas de los Altos de Chiapas consiste en gran parte de especies de plantas con mayor distribución y abundancia en la región. Además, estas especies son reconocidas ampliamente por la población en general. La ponencia intenta rechazar el mito que este conocimiento representa "secretos centenarios" (Caplan 2000: 1) en la posesión exclusiva de curaderos, shamanes, y otras especialistas médicas.

\section{Desarrollo}

Presentamos en seguida datos recolectados en dos proyectos etnobotánicos llevado acabo por los autores y sus colaboradores en los Altos de Chiapas durante los últimos ocho años (ver mapa, Figura 1)

Figura 1 : Area de estudio de los proyectos etnobotánicos en los Altos de Chiapas, México

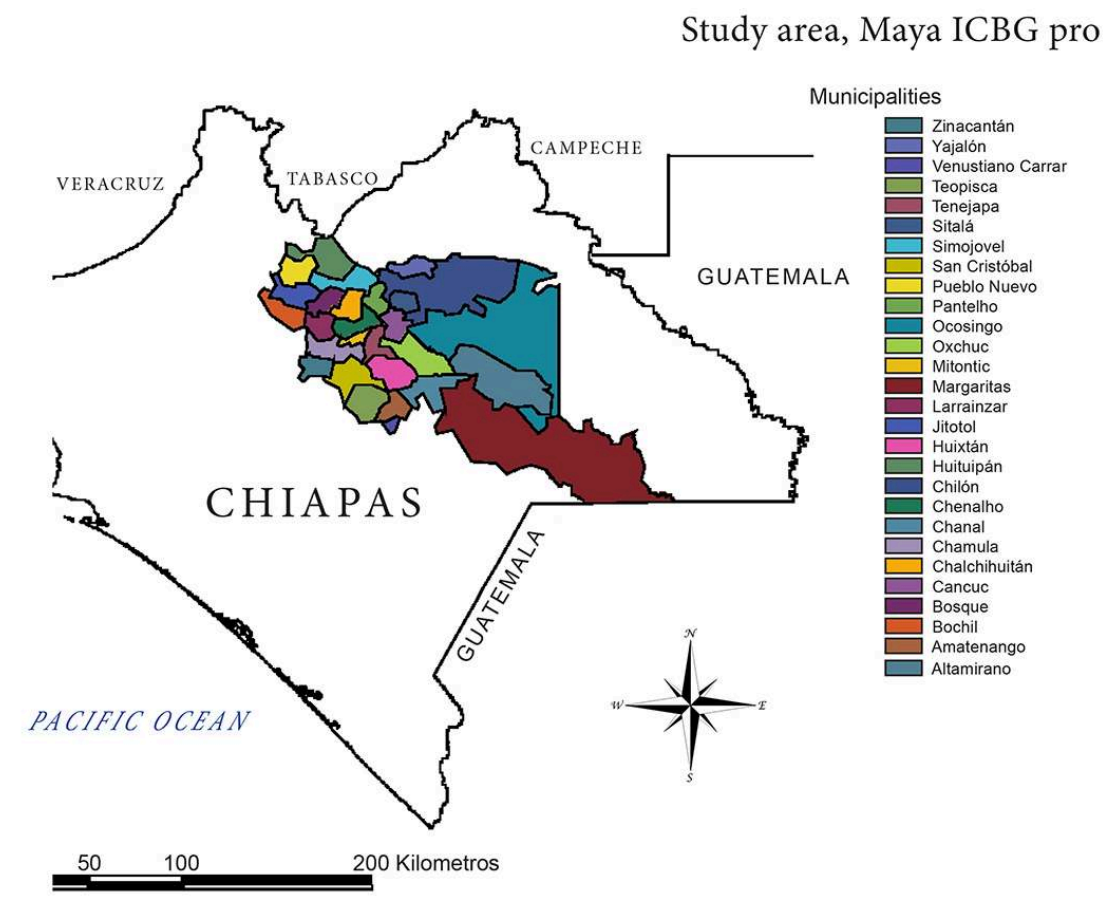

El primer proyecto fue conducido entre los años 1985-1989 en colaboración con Xavier Lozoya, Mariana Meckes, Ma. Luisa Villarreal, y Jorge Tortoriello, entonses investigadores del Centro de Investigación del Sur (IMSS) en Xochitepec, Morelos, el 
botánico Dennis E. Breedlove, entonses de la Academia de Ciencias de California, el antropólogo Roberto M. Laughlin de la Institución Smithonian, y muchos colegas mayas. El proyecto, nombrado el « Programa de Cooperación sobre Medicina Indígena Tradicional y Herbolaria » (PROCOMITH) enfocó en un estudio del sistema etnomédica de los tzeltales y tzotziles de los Altos de Chiapas. Como parte del proyecto se realizó 6957 colectas que representan unas 1580 especies. Todas estas epecies supestamente tienen valor medicinal y alrededor de 600 especies muestran una disbribución general en la región. Unas 100-200 epecies están conocidas ampliamente y son las más importantes en el tratamiento de las enfermades reconocidas por las comunidades mayas de los Altos.

5 El segundo proyecto, designado el ICBG-Maya (por su siglas en inglés, the Maya International Cooperative Biodiversity Group), realizó colectas botánicas como parte de un estudio de la biodiversidad de los Altos de Chiapas en colaboración con investigadores del Colegio de la Frontera Sur (ECOSUR) en San Cristóbal de Las Casas y un grupo de asistentes mayas entrenados en la metodología botánico de campo. Este proyecto enfocó en llevar acabo colectas de la flora general no solamente de plantas útiles. Durante los meses de julio a noviembre de 1999, realizamos 6324 colectas que representan alrededor de 1368 epecies. Además, el proyecto promovió la formación de jardines etnobotánicos en las comunidades mayas y se llevó acabo estudios intensivos sobre las especies más utlizadas en la preparación de remedios herbolarios.

\section{Resultados}

6 Antes que presentamos los datos de los dos estudios mencionados arriba, tenemos que hablar brevemente sobre la diversidad botánica de los Altos de Chiapas. Breedlove (1986), en su famoso Listados de la flora de Chiapas presenta un inventario de 6823 epecies en 1735 géneros en 198 familias. Estas cifras son para el estado en general. Los herbarios de ECOSUR en San Cristóbal de Las Casas tienen registrado un inventario de 4115 especies en 1419 géneros en 203 familias. Es importante señalar que estas especies son principalmente de la flora de los Altos. Chiapas muestra un nivel de biodiversidad de plantas vasculares que cuenta entre las más altas del mundo (Barthlott et al. 1996).

El proyecto PROCOMITH hizo colectas durante un periodo de 15 meses en 9 municipios, entrevistando más que 350 colaboradores mayas sobre la medicina tradicional. De las 1580 epecies representadas en las colectas, 204 fueron colectadas varias veces ( 7 a 20 colectas cada especie). Estas 204 especies formaron una colección de espécimenes presentadas en encuestas etnomédicas con 126 colaboradores adicionales en 14 municipios (Aguacatenango, Amatenango del Valle, San Juán Cancuc, Chanal, Chalchihuitán, San Juán Chamula, San Pedro Chanalhó, San Andrés Larráinzar, Huixtán, Mitontic, Oxchuc, Pantelhó, Tenejapa, y Zinacantán). El base de datos de estas encuestas cuenta con más de 30,000 « records ». Algunos resultados de estes estudios están publicados en Berlin E. y Berlin B. (1996), Berlin B. y Berlin E. (1998), Berlin et al. (1990) y Meckes et al. (1995).

8 El proyecto ICBG-Maya hizo colectas generales en cuarto municipios de los Altos en julio a noviembre de 1999. El reconocimiento botánio resultó en 6324 colectas que representan 1368 epecies. Colectores mayas recibieron entrenimiento en la metodogía de hacer colectas botánicas con instrucciones de colectar cualquier especie que encontraron con flor o fruta (es decir, en condiciones que permite una determinación 
botánica hasta el nivel de especie). Algunos resultados de estes estudios están publicados en Berlin et al. (1999) y Berlin et al. (2001).

Unas 167 especies en las colectas del proyecto ICBG-Maya están representadas por 9 hasta 88 colecciones cada una. Se puede mencionar como ejemplos de especies con el mayor número de colectas como Lantana camara, Acacia angustissima, Monnina xalapensis, Crusea calocephala, Cuphea aequipetala, y Borreria laevis. Especies representadas por 9 colectas son, por ejemplo, Sida barclayi, Lobelia cardinalis, Salvia cinnabarina, Acacia pennatula, y Hybanthus verbenaceus.

Si comparamos las especies de más frecuencia del proyecto ICBG-Maya (colecciones generales) con las de más frecuencia del proyecto PROCOMITH (especies con valor medicinal), se nota que $72 \%$ de las especies medicinales son las mismas especies o especies en el mismo género. Si selecionamos las 54 especies con colectas $>19$ en el base de datos del ICBG-Maya, se puede observar que más que $89 \%$ son las mismas incluidas en el base de datos de especies más comunes en el proyecto PROCOMITH (vease Tabla 1).

\section{TABLA 1 : Comparación de las 54 epecies de colecta más frecuente}

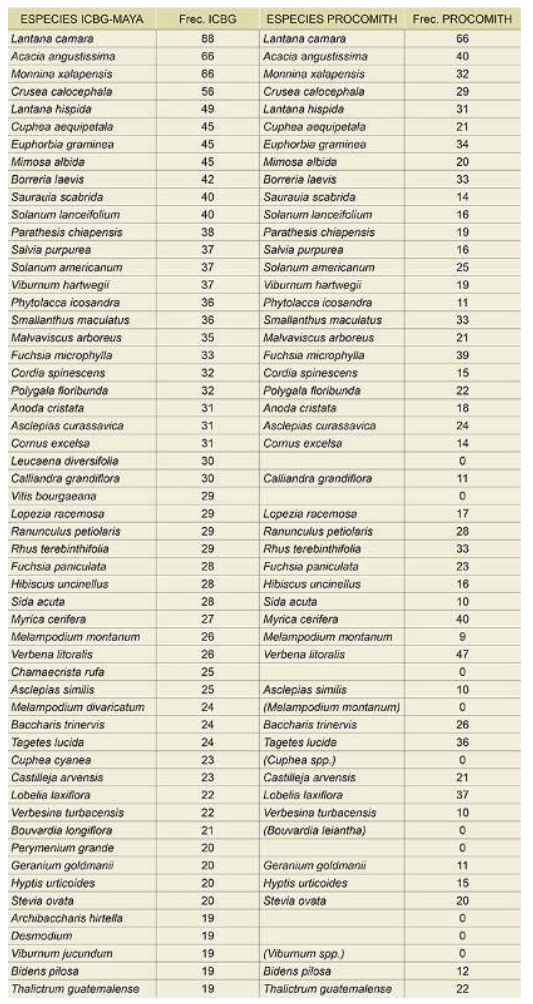

11 ( $\geq 19$ colecciones cada especie) en el proyecto ICBG-Maya con las especies medicinales más comunues en el proyecto PROCOMITH

Adicionalmente, el proyecto ICBG-Maya promovió el establecimiento de 8 jardines botánicas en 4 muncipios (Chenalhó, Tenejapa, Zinacantán, y Oxchuc) y colectó información etnomédica sobre la formularia maya (ingredientes, preparación, y administración de remedios hierbales). El promedio de especies sembradas en los jardines selecionadas por miembros de cada comunidad es alrededor de 450 en no menos de 100 familias botánicas. De las especies representadas por $\geq 5$ ejemplares en los jardines etnobotánicos, 77 \% están representadas en las colectas de más frecuencia en 
el proyecto ICBG-Maya. De las especies mencionadas $\geq 4$ veces en las encuestas etnomédicas sobre la formularia maya, 50 \% corresponden con las especies de colecta más frecuente por el ICBG-Maya.

Como una indicación final de la relación de la frecuencia de colección y la flora medicinal podemos dividir las colecciones del ICBG-Maya en dos clases-- las especies que muestran una alta frecuencia de coleccción $(\geq 8)$ y las especies que muestran una baja frecuencia de colección $(\leq 2)$. Luego, dividimos estas dos grupos según el supuesto valor medicinal de las especies en el base de datos de PROCOMITH. Se puede ver los resultados en la Tabla 2 .

Tabla 2 : Relación de frecuencia de colecta de especies del proyecto ICBG-Maya con su supuesto valor medicinal (PROCOMITH) Colectas Proyecto ICBG Maya

\begin{tabular}{|c|c|c|}
\hline & $\begin{array}{c}\text { Frec. Col. Alta } \\
(n=190 \mathrm{spp} . \geq 8)\end{array}$ & $\begin{array}{l}\text { Frec. Col. Baja } \\
(n=725 \text { spp. } \leq 2)\end{array}$ \\
\hline+ & 172 & 116 \\
\hline \multicolumn{3}{|l|}{$\begin{array}{c}\text { Valor medicinal } \\
\text { (según datos de PROCOMITH) }\end{array}$} \\
\hline- & 18 & $\begin{array}{r}609 \\
n=915 \text { spp. } \\
\text { Chio-square }=388 \\
P<.001\end{array}$ \\
\hline
\end{tabular}

En fin, los resultados indicados en nuestro analysis de los bases de datos de los dos proyectos indican claramente que las especies que tienen una extensión ecológica muy amplia y que se encuentran facilimente por razones de su abundancia ( la flora común ») son las mismas especies selecionadas por las comunidades mayas como las plantas medicinales de más importancia en su herbolaria ( « la flora medicinal »).

Más relevante, estas especies comunes con gran valor medicinal son conocidas ampliamente por la población general de las comunidades mayas de región. Presentamos abajo datos de enquestas etnomédicas recolectadas en 8 municipios tzotziles del proyecto PROCOMITH (los datos de los 6 muncipios de habla Tzeltal no se presenta ahora). Analizando las respuestas sobre el uso medicinal de las 41 especies de colecta más frecuente en el proyecto ICBG-Maya, y referiéndose a datos etnomédicos sobre estas especies en la base de datos de PROCOMITH, podemos notar que hay una concordancia promedio de $70 \%$ entre los datos de los 8 municipios sobre su uso medicinal (vease Tabla 3). Estos datos confirman que el conocimento de los usos medicinales de las especies más comunes es compartido entre las comunidades mayas. Este conocimiento constituye «la medicina maya popular» en el mismo sentido que hablamos de "la medicina mexicana popular», conocimiento que tiene una amplia extension en la población general. 
Tabla 3 : Concordancia de conocimiento de uso medicnal en 8 municipios tzotziles de los Altos de Chiapas para las epecies de colecta más frecuente

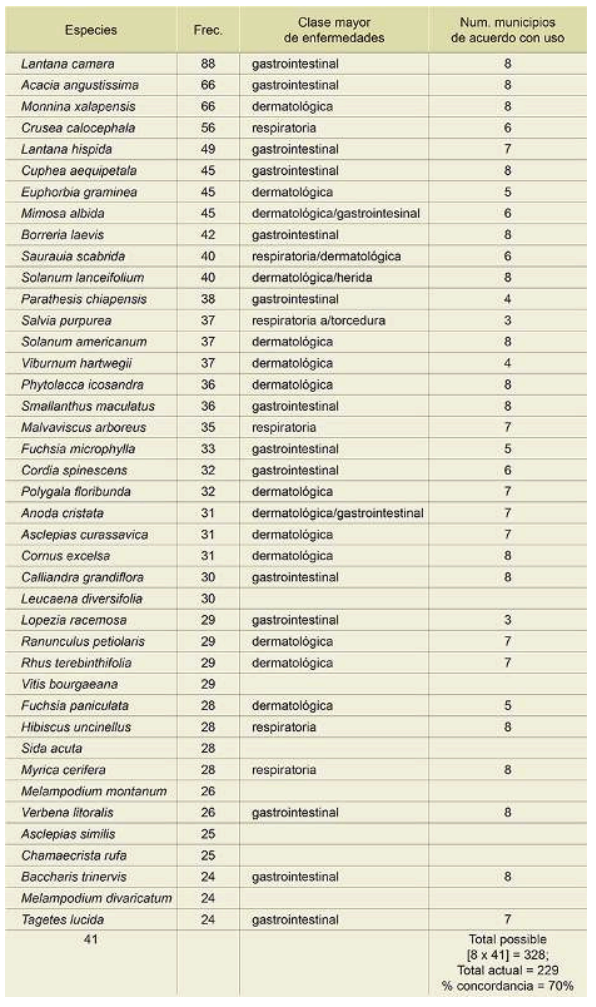

\section{Conclusiones}

Hay un mito todavía que la medicina indígena tradicional es el sistema médica representada por el conocimento controlado por unos pocos especialistas, llamadas comunmente « curanderos », « shamanes », o « médicos tradicionales ». Es cierto que el conocimiento de estas especialistas no está compartido con la población en general y en muchos casos es considerado información secreto. Los medicos tradicionales tzeltales y tzotziles utliza su conocimiento especial en el tratamiento de enfermedades que normalmente tienen una etiología o manifestación extra-ordinaria.

De hecho, el enfermo normalmente busca un curandero solamente en casos de enfermedades que él mismo o un familiar no se puede curar con plantas medicinales. Aunque el shaman puede usar plantas medicinales en la curación - plantas conocidas por la población en general- el poder curativo de su intervención es através de su conocimiento de rituales especiales que incluye rezos, visiones, sacrificios, y comunicación con entidades espirituales.

18 Además, hay varios clases de curanderos especiales todos con nombres específicas (vease j'ilol o jpoxtawanej 'shamán', jpik ch'ich' 'pulsador', jch'abajom 'rezador a los cerros'). En cambio, no existe un término especial para 'hierbero' en Tzeltal o Tzotzil. Las personas que tienen conocimiento de plantas que sobrepasa el nivel de la comunidad en general se puede designar con una frase descriptiva como ja' mach'a ya sna' sba poxil wamal 'el/ella que tiene conocimiento de plantas medicinales'.

19 Desgraciadamente los que creen que la herbolaria indígena es principalmente «secreto» o «oculto», restringuido al dominio de los médicos especialistas 
tradicionales, han concluido que los científicos quienes intentan estudiar la herbolaria en actualidad "pretenden robar" este conocimiento por fines lucrativos, robo designado como « la biopiratería » (Caplan 2000 : 1). Esta visión romántica y errónea es uno de los factores principales que prohiben estudios profundos de la medicina indígena popular con fines de confirmar la bioactivad de especies comunes, su toxicidad, control de calidad, certificación, producción y comercialización justa. En lugar de apoyar las comunidades índias, la visión del romántico es una visión que pone limitaciones serveras en el desarrollo económico de las mismas comunidades indígenas -comunidades que andan en poz de alternatives económicas que se puede derivar del uso justo y productivo de su conocimiento popular.

\section{Propuestas}

Que el comité ejecutivo del Primer Congreso Latinoamericano de Herbolaria comisiona un grupo técnico con el cargo de clarificar las grandes diferencias entre el conocimiento de «la medicina indígena popular» de la población en general y «la medicina secreta» de los médicos tradicionales especialistas, personas que curan atraves de poderes sobre-naturales. Además que el grupo técnico elabora un "white paper " sobre estas grandes diferencias que puede tener distribución amplia en la comunidad científica, los medios de comunicación, y en el Internet, abriendo el camino para las investigaciones científicas de la medicina indígena popular que se merece.

\section{BIBLIOGRAPHY}

Barthlott W., Lauer W. \& Placke A. 1996 - A global distribution of species diversity in vascular plants: towards a world map of phytodiversity. Erdkunde $50: 317-327$.

Berlin E.A. \& Berlin B., con contribuciones por Lozoya X., Ricárdez J.R., Breedlove D.E., Meckes M., Tortoriello J., Villarreal M., Rodríguez G., Laughlin R.M. \& Maffi L.1996 - Medical Ethnobiology of the Highland Maya: The Gastrointestinal Diseases. Princeton, N. J., Princeton University Press.

Berlin E.A. y Berlin B.1998 - Enciclopedica Médica Maya. Cuatro-lingual CD ROM sobre la medicina maya de los Altos de Chiapas. San Crisóbal de Las Casas, Chiapas, Mexico, El Colegio de la Frontera Sur.

Berlin B., Berlin E.A., Breedlove D.E., Duncan T., V. Jara, Astorga, Laughlin R.M., and T. Velasco C. 1990 - La Herbolaria Médica Tzeltal-Tzotzil en los Altos de Chiapas, México: Un Ensayo Preliminar Sobre Las Cincuenta Especies Botánicas de Uso Más Frecuente.. Tuxtla Gutiérrez, Chiapas, Mexico: Instituto Chiapaneco de Cultura.

Berlin, B., Berlin E.A., Fernandez Ugalde J.C., Garcia Barrios L., Puett D., Nash R. \& GonzalezEspinoza M. 1999 - The Maya ICBG: Drug Discovery, Medical Ethnobiology and Alternative Forms of Economic Development in the Highland Maya Region of Chiapas, Mexico. Pharmaceuical Biology 37 (4) : 27-144. 
Berlin B., Berlin E.A., L. García B., M. González E., Puett D. \& Nash R. 2001 - Etnobotánica Médica y Dessarrollo Sustentable: El Caso del ICBG-Maya en Los Altos de Chiapas ». In : B. Rendón A., S. Rebollar D., J. Caballero N. \& M-A Martínez A. (Ed.) Plantas, Cultural y Sociedad: Estudios Sobre la Relación entre Seres Humanos y Plantas en los Albores del Siglo XXI. . México, D. F., Universidad Autónoma Metropolitana, Unidad Iztapalaga y Secretaría del Medo Ambiente, Recursos Natruales y Pesca : 221-258.

Breedlove D. E. 1986 - Listados Florísticos de México : IV Flora de Chiapas. México, D. F., Instituto de Biología, UNAM.

Caplan A. 2000 - Bio-colonialism and Maya Medicine in Chiapas, » Witness for Peace Newsletter (Winter 2000, p. 1), citada en Lisa J. Adams, « Herbal drug war erupts in Mexico, » Associated Press, April 2001.

Meckes M., Villarreal Ma. Luisa, Tortoriello J., Berlin B. \& Berlin E.A. 1995 - A Microbiolgoical Evaluation of Medicinal Plantas used by the Maya People of Southern Mexico. Phytotherapy Research $9: 244-250$

\section{ABSTRACTS}

This essay draws a definitive distinction between special or secret knowledge and popular [folk or common] knowledge of the medicinal plant species that form the core of the empirical herbal medicine of the Tzeltal and Tzotzil of the Chiapas Highlands. We intend to demonstrate that the Maya herbolaria consists principally of the common flora of the region. In addition, these species are recognized by the general population, not only by specialists or individuals who identify as traditional doctors. We can conclude that the body of Tzeltal-Tzotzil herbal knowledge is, then, the folk medicine of the Maya in the same sense as we may speak of Mexican folk medicine. The herbolaria of the Maya does not consist of sacred information nor secret knowledge. The system is public and merits study by anthropologists, ethnobotanists, students of ethnomedicine, and laboratory analysis, always in close collaboration with the Mayan communities, with regard to methods of preparation and administration, bioactivity, toxicity, and quality control.

Este ponencia se hace distinción definitiva entre el conocimiento especial o secreto $\mathrm{y}$ el conocimiento popular de las especies de plantas medicinales que forman la base de la herbolaria empírica de los tzeltales y tzotziles de los Altos de Chiapas. Pretendemos demostrar que la herbolaria maya consiste principalmente de la flora común de la región. Además, estas especies están reconocidas por la población en general, no solamente por unas especialistas o individuos que se identifican como médicos tradicionales. Podemos concluir que la herbolaria tzeltal-tzotzil es, entonces, la medicina maya popular en el mismo sentido que podemos hablar de la medicina mexicana popular. La herbolaria de los mayas no consiste en información sagrada ni conocimiento secreto. El sistema es público y merece estudios antropológicos, etnobotánicos, etnomédicos, y de laboratorio, siempre en estrecha colaboración con las comunidades mayas, sobre métodos de preparación y administración, bioactividad, toxicidad, y control de calidad.

Cet essai établit une distinction définitive entre la connaissance spéciale ou secrète et la connaissance populaire (ou commune) des plantes médicinales, qui forment le noyau de la phytothérapie empirique des Tzeltal et Tzotzil des Hautes-Terres du Chiapas. Nous démontrons que l'herbolaria maya est principalement composée de la flore commune de la région. De plus, ces espèces sont reconnues par la population générale, et pas seulement par des spécialistes ou des personnes qui s'identifient comme médecins traditionnels.

Nous pouvons en conclure que le corpus des connaissances tzeltal-tzotzil sur les plantes 
constitue la médecine populaire des Mayas, dans la même façon que nous pouvons parler de médecine populaire mexicaine.

L'herbolaria des Mayas ne comporte pas d'informations sacrées ni de connaissances secrètes. Le système est public et mérite d'être étudié par des anthropologues, des ethnobotanistes, des étudiants en ethnomédecine et des laboratoires, toujours en étroite collaboration avec les communautés mayas, en ce qui concerne les méthodes de préparation et d'administration, la bioactivité, la toxicité et le contrôle de la qualité.

\section{INDEX}

Keywords: medicinal plants, secret or common knowledge, folk medicine, Mexico, Mayas, ethnobotany

Palabras claves: plantas medicinales, conocimiento secreto, medicina popular, México, Maya, etnobotánica

Mots-clés: plantes médicinales, connaissances secrètes ou publiques, médecine populaire, Mexique, Mayas, ethnobotanique

\section{AUTHORS}

\section{BRENT BERLIN}

Laboratorios de Etnobiología - Departamento de Anthropologia, Universidad de Georgia - Athens, Georgia 30302 EU - obberlin@gmail.com

\section{ELOIS ANN BERLIN}

Laboratorios de Etnobiología - Departamento de Anthropologia, Universidad de Georgia - Athens, Georgia 30302 EU - eaberlin890@gmail.com 